\title{
Migrantes colombianos en Chile: Tensiones y oportunidades en la Articulación de una Historia Personal *
}

\section{Colombian migrants in Chile: Tensions and opportunities in the articulation of life stories}

Recepción: 11 Junio 2017 | Aprobación: 14 Agosto 2017

\author{
Andrea Rihm Bianchia ${ }^{\mathrm{a}}$ \\ Pontificia Universidad Católica de Chile, Chile \\ ORCID: http://orcid.org/0000-0003-1251-9857 \\ Dariela Sharim Kovalskys \\ Pontificia Universidad Católica de Chile, Chile
}

a Autor de correspondencia. Correo electrónico: arihm@uc.cl

Para citar este artículo: Rihm, A., \& Sharim, D. (2017) Migrantes colombianos en Chile: Tensiones y oportunidades en la Articulación de una Historia Personal. Universitas Psychologica, 16(5), 1-15. https:// doi.org/10.11144/Javeriana.upsy16-5.mcto

\section{RESUMEN}

Este artículo presenta y discute los resultados de un estudio cualitativo desarrollado en Chile, que exploró y analizó las significaciones atribuidas por migrantes colombianos adultos a sus experiencias. Se utilizaron relatos de vida y creaciones visuales para producir datos. Los resultados evidenciaron la pluralidad de significaciones en torno a la migración, así como el proceso continuo de elaboración y evaluación que supone dar sentido a la experiencia, pues esta revela la naturaleza contingente de la identidad, el estilo de vida y las prácticas culturales. Los participantes tendieron a significar positivamente su experiencia migratoria, pero evidenciaron la presión por salir adelante con base en sus propios recursos, caracterizando la migración como un proyecto inherentemente personal, reflejando tendencias actuales a la individualización social.

Palabras clave

experiencias de inmigración; relatos de vida; transformaciones culturales; Chile; Colombia.

\footnotetext{
ABSTRACT

This article presents and discusses the results of a qualitative mixedmethod study conducted in Chile aimed to explore the subjective experiences and meanings attributed by a group of adult Colombian immigrants to their migratory experience. Life stories and artworks were used to produce data. The study found that migratory trajectories are signified in plural ways. Immigration demanded a continuous process of sense-making by the participants, showing the contingent nature of identity, life style and cultural practices. Participants tended to signify positively their migration experiences, although felt a strong pressure to succeed and get ahead, relying mostly on their own personal resources. Therefore, the results showed that migration is experienced in an inherently individual way, reflecting the individualization present in culture.

Keywords

immigration; experiences; life stories; cultural transformations; Chile; Colombia.
} 


\section{Introducción}

Las migraciones son uno de los fenómenos definitorios de nuestro tiempo, marcado por la fluidez y los desplazamientos (Arfuch, 2010; Bauman, 2003; Esses, Deaux, Lalonde, \& Brown, 2010). Los aproximadamente 214 millones de inmigrantes en el mundo constituyen alrededor de un $3.1 \%$ de la población, y representan la mayor cantidad de personas viviendo fuera de sus países de origen en la historia (Esses et al., 2010; Organización Internacional para las Migraciones, 2013).

En Chile, la migración no es un fenómeno nuevo. Entre la última década del siglo XIX y la primera del siglo XX, la población migrante alcanzó sus niveles históricos más altos $(4.1 \%$ en 1907), debido, por una parte, a las políticas estatales implementadas desde la segunda mitad del siglo XIX, orientadas a atraer migrantes europeos que aportaran capital social y cultural, bajo la premisa de que un incremento en el volumen de la población impulsaría el progreso económico; y por otra, a la atracción que ejerció la minería en el norte del país (Cano \& Soffia, 2009; OIM, 2011; Ravanal, 2005).

Sin embargo, desde los años veinte, las migraciones experimentaron un declive significativo hasta alcanzar su nivel histórico más bajo en 1982, bajo dictadura, cuando representaba solo un $0.75 \%$ de la población. En 1992, los migrantes no llegaban al 1\%, pero en el año 2002 constituían un 1.2\%, en el 2014 superaban el 2\% (aproximadamente 411 000 personas), y durante el 2015, de acuerdo con cifras de la Encuesta de Caracterización Socioeconómica Nacional (CASEN), serían 465 319 personas, correspondientes al 2.7\% de la población nacional. Las últimas estimaciones constituyen la mayor cifra registrada en Chile desde la segunda mitad del siglo XX, y por primera vez en décadas la cantidad de inmigrantes podría estar superando la de chilenos emigrantes (Courtis, 2011; Ministerio del Interior, 2010, 2016; OIM, 2011; Solimano \& Tokman, 2007). Este ascenso se explicaría por la rigidez de las políticas migratorias de los países desarrollados y por la estabilidad económica alcanzada por Chile en las últimas décadas, que ha contribuido a posicionarlo como un destino atractivo en Latinoamérica para la búsqueda de mejores condiciones de vida (Cárdenas \& Yáñez, 2012; OIM, 2011; Ravanal, 2005). En este contexto, las migraciones actuales, a diferencia de las migraciones históricas, son mayoritariamente sur-sur, y autónomamente dirigidas por los propios migrantes.

Según la Encuesta CASEN 2015, elaborada por el Ministerio de Desarrollo Social, el grupo mayoritario de inmigrantes en Chile proviene de Perú (30\%), seguido por personas provenientes de Colombia (13.3\%), y Argentina (11.9\%). El ascenso de la migración colombiana ha sido particularmente marcado desde el año 2000, aumentando en un 394\% entre el 2005 y el 2014, y continuó en alza hasta sobrepasar la migración argentina, que hasta el 2013 representaba el segundo colectivo con mayor presencia (Ministerio de Desarrollo Social, 2016).

$\mathrm{Si}$ bien este ascenso sigue siendo un tema poco investigado, se ha planteado que se relacionaría con cambios en la situación económica y política en Colombia, en la que bajas en la inversión y producción se tradujeron en un aumento significativo del desempleo (OIM, 2011; Soler, 2004) y, en segunda instancia, con condiciones de violencia en Colombia que habrían incidido en el aumento del flujo de migrantes pidiendo refugio, especialmente cuando su calidad de refugiados no les es reconocida en otros países de la región (OIM, 2011).

Estos cambios en el perfil migratorio de Chile, ocurridos velozmente y en ausencia de un marco legislativo apropiado (Doña Reveco \& Mullan, 2014), suponen desafíos sociales importantes y evidencian la necesidad de investigar para desarrollar políticas públicas adecuadas (Cano \& Soffia, 2009; Martínez, 2005; Ravanal, 2005). Para lograrlo, es necesario abordar multidimensionalmente la migración, incluyendo su dimensión subjetiva, que contribuiría a desafiar la construcción de sobregeneralizaciones y estereotipos (De Fina, 2003). Así, es relavente aproximarse al estudio de las experiencias subjetivas de migración desde una perspectiva singular, que rescate la particularidad de 
cada caso, indagando en su profundidad y articulándolo con lo histórico, social y cultural.

Un tema fundamental al considerar las experiencias subjetivas de los migrantes, y su impacto en las historias de vida, es la identidad. De hecho, se ha planteado que, trascendiendo las diferencias de cada proceso migratorio, estos compartirían una serie de características nucleares; entre ellas, el cuestionamiento de la identidad y la experiencia de duelo (Akhtar, 1995; Grinberg \& Grinberg, 1996).

La inmigración pondría en marcha un proceso de cambio personal, en el que la pregunta por la identidad: "iquién soy?" se transformaría en "iquién soy acá?", donde la experiencia de "simplemente vivir" se convertiría en "vivir en un lugar" (Akhtar, 2014). Así, la migración visibilizaría la diferencia y la cultura de un modo tal, que esta perdería su carácter de "telón de fondo" en el que nos sumergimos cotidianamente hasta que se vuelve transparente (Boulanger, 2004), instalando una fisura en la historia de vida, evidenciando el carácter contingente de la identidad y revelando la apertura del destino y la necesidad de un proceso de continua definición y redefinición respecto al sentido de sí mismo y de pertenencia (Arfuch, 2010; De Fina, 2003).

Dados estos antecedentes, la psicología clínica ha tendido a enfatizar las pérdidas y duelos que conlleva la inmigración, considerándose hasta tal punto entrelazados, que se ha planteado que "cuando una persona emigra, empieza un proceso de duelo" (Lobban, 2006, p. 75). Se ha destacado la complejidad del desafío que enfrentan los inmigrantes, de elaborar la pérdida de la cultura de origen manteniendo cierto apego a esta, simultáneamente desarrollando nuevos vínculos y relaciones en el país de llegada (Akhtar, 1995; Arfuch, 2010, Camilleri \& Malewska-Peyre, 1997; De Fina, 2003; Grinberg \& Grinberg, 1996; Lijtmaer, 2001).

Así también, se ha planteado que la necesidad de mantener cierta congruencia identitaria, pertenencia y valorización de sí llevaría a los migrantes a desplegar, consciente o inconscientemente, estrategias identitarias para evitar o reconciliar las contradicciones, tanto como para defenderse de posibles amenazas y evitar la estigmatización y la desvalorización (Camilleri \& Malewska-Peyre, 1997). Berry (2005, 2007) extendió el concepto de estrategias identitarias más allá del plano individual, y propuso la noción de estrategias de aculturación. Esto, conceptualizando la aculturación como el proceso de transformación --cultural y psicológico- que ocurre a nivel grupal e individual a partir del contacto sostenido entre culturas diferentes. Así, las estrategias de aculturación serían actitudes y conductas que se despliegan en el encuentro intercultural, y que en el caso de los migrantes, dependiendo de la valoración y búsqueda de relaciones con el otro grupo y de la importancia atribuida a mantener la herencia e identidad cultural, podrían seguir cuatro grandes líneas: integración, asimilación, separación y marginalización (Berry, 2005, 2007).

La noción de aculturación ha sido criticada por asumir que los procesos psicológicos que operan en ella tendrían una estructura similar en personas con orígenes culturales diversos (Hermans, 2001). Aunque Berry (2005) no establece matices respecto a orígenes culturales, sí destaca que los migrantes no siempre pueden desplegar sus estrategias de preferencia, pues el contexto cultural es determinante de sus posibilidades de acción, y puede reforzar la elección de algunas estrategias por sobre otras. En este sentido, plantea que el proceso de aculturación conlleva conflictos, necesidad de negociación y estrés, aunque considera que a menudo termina en alguna forma de acomodación mutua.

Por otra parte, las perspectivas que asocian ineludiblemente inmigración con duelo han sido criticadas por considerar que, al enfatizar las pérdidas, no problematizan suficientemente las relaciones de los migrantes con su lugar de origen ni los sentimientos de pertenencia en relación con este, al mismo tiempo que tampoco considerarían suficientemente las oportunidades que la migración conlleva. Así, se ha planteado la necesidad de renovar el enfoque para que dé cuenta del modo en que las transformaciones culturales han afectado las formas en que los migrantes actuales construyen y significan 
sus trayectorias migratorias (Beltsiou, 2016; Bobowik, 2013; González, 2016).

Esto último es particularmente relevante en el contexto cultural actual, marcado por el declive de la relevancia de los referentes sociales y colectivos como espacios donde encontrar sentido, y el incremento de la responsabilidad individual respecto a la propia trayectoria de vida (Bauman, 2003; Lipovetsky \& Charles, 2006). Así, cabe hipotetizar que la mayor fluidez y globalización cultural en la que vivimos impacta en el modo en que los migrantes significan sus trayectorias, cuestionando, quizás, nociones más tradicionales respecto a que "los inmigrantes son quienes se van porque tienen que hacerlo" (Behar, 2013, p. 5).

Este artículo espera aportar en tal sentido, presentando y discutiendo parte de los resultados de una tesis doctoral cuyo objetivo fue aproximarse al estudio de la experiencia subjetiva de migración de un grupo de colombianos en Chile. Desde una perspectiva singular, que se articula con lo histórico, social y cultural, se buscó dar espacio a la expresión tanto de los desafíos, conflictos y problemas asociados a la experiencia migratoria, como a las oportunidades de apertura y desarrollo que esta implica, siguiendo la voz de los propios migrantes. Las preguntas que guiaron el estudio fueron ¿Cómo construyen sentido los migrantes en sus experiencias de migración? ¿Qué estrategias despliegan para enfrentar la experiencia? ¿Cómo articulan la experiencia de migración en el marco de su biografía? ¿Cómo impacta la migración en el desarrollo de la identidad?

\section{Método}

\section{Diseño}

Desde un enfoque cualitativo, biográfico, se utilizaron herramientas mixtas, visuales y verbales, para conocer los significados y experiencias subjetivas desde el propio marco de referencia de los migrantes (Cornejo, 2006; Flick, 2004). Esta combinación buscó generar espacios de expresión de la naturaleza compleja y multifacética del fenómeno (Moran-Ellis et al., 2006). Esto, pues aunque los relatos de vida ofrecen una aproximación privilegiada a la subjetividad e identidad desde una perspectiva culturalmente situada, suponen la capacidad de los sujetos de formular y comunicar verbalmente sus experiencias, lo que no siempre es posible. Las obras visuales, por su parte, permitirían evocar emociones y expresar experiencias difícilmente formulables de otro modo (Reavey \& Johnson, 2008; Weber, 2008).

\section{Participantes}

Se seleccionaron gradualmente 14 migrantes colombianos adultos (siete hombres y siete mujeres), siguiendo la lógica del muestreo teórico (Flick, 2004). El contacto fue a través de informantes claves y se complementó con un procedimiento de "bola de nieve" (Patton, 2002). Se realizó una búsqueda de participantes de variados niveles socioeconómicos, puesto que la inmigración colombiana abarca diversos sectores laborales, sociales y urbanos.

La mayoría de los migrantes que llegan a Chile, lo hacen en edad laboral activa. Más del $75 \%$ tienen entre 20 y 50 años (Ministerio del Interior, 2010, 2016). Por esto, se contactaron participantes en ese rango etario. Todos los participantes vivían y trabajaban en la ciudad de Santiago, se encontraban en Chile desde hace al menos dos años (salvo en el caso de una participante que durante el proceso de entrevistas reveló que, en realidad, llevaba un año y algunos meses), y habían migrado voluntariamente durante su adultez. Todos habían llegado a Santiago a partir del año 2000. Los participantes no eran receptores de servicios de organizaciones de ayuda a migrantes ni participaban activamente en alguna organización comunitaria relacionada.

Puesto que las migraciones forzadas especialmente en condiciones de violenciaadquieren un carácter particularmente complejo, que se manifiesta en mayores dificultades de elaboración y en mayores efectos negativos en la identidad (Grinberg \& Grinberg, 1996; Volkan, 
2004), se estableció como criterio de exclusión que los migrantes hubieran entrado a Chile en calidad de refugiados.

TABLA 1

Identificación de los Participantes

\begin{tabular}{|c|c|c|c|c|}
\hline Pseudónimo & Edad & $\begin{array}{c}\begin{array}{c}\text { Años en } \\
\text { Chile }\end{array} \\
\end{array}$ & $\begin{array}{l}\text { Ciudad de } \\
\text { Origen }\end{array}$ & Ocupación \\
\hline Camilo & 26 & 4 & Bogotá & $\begin{array}{l}\text { Ingeniero en } \\
\text { Telecomunicaciones }\end{array}$ \\
\hline Fernando & 31 & 2.5 & Ammenia & Junior Administrativo \\
\hline Pablo & 32 & 2 & & Ejecutivo de Ventas \\
\hline Afforso & 33 & 10 & San Onofie & Dentista \\
\hline Jaime & 34 & 14 & Cali & Profesional Cs. Sociales \\
\hline Manolo & 44 & 9 & Buenaventura & Feriante \\
\hline Victor & 48 & 7.5 & Cartagena & Ingeniero Informático \\
\hline Ana Laura & 29 & 4 & Bucaramanga & Diseñadora Gráfica \\
\hline Beatriz & 30 & 2 & Valle del Cauca & $\begin{array}{l}\text { Recepcionista } \\
\text { (Ingeniera Industrial) }\end{array}$ \\
\hline Lucia & 36 & 2.5 & Pereira & $\begin{array}{l}\text { Vendedora de } \\
\text { Colaciones }\end{array}$ \\
\hline Marisol & 37 & 5 & & Higienista Dental \\
\hline Gracia & 42 & 3.5 & Bogotá & Vendedora \\
\hline Alejandra & 42 & 7.5 & Bucaramanga & Abogada \\
\hline Nancy & 46 & 1 & & Asesora del Hogar \\
\hline
\end{tabular}

Dispositivos de Producción de Datos

Se utilizaron relatos de vida temáticos y creación de obras visuales. Los relatos de vida temáticos son narraciones que realizan los sujetos de un aspecto de su vida, que permiten recuperar los significados y sentidos atribuidos a las experiencias vividas (Cornejo, 2006; De Gaulejac, 1996, Sharim, 2001). En particular, se utilizó la consigna: "cuéntame la historia de tu vida en Chile".

Las obras visuales son creaciones personales realizadas por los participantes con el objetivo de representar algún aspecto significativo de su experiencia. La consigna utilizada para obtener estas obras fue: "te voy a pedir que con los materiales disponibles, crees una imagen que evoque o represente una experiencia que para ti sea significativa en relación a tu experiencia de migrar". En cada ocasión se ofrecieron variados materiales artísticos. No se entregaron lineamientos respecto a qué tipo de obra realizar para promover la auto-expresión y el sentido de autoría de los participantes, puesto que la elección de materiales y medios constituye una primera forma de posicionamiento subjetivo (Moon, 2010). Del total de participantes, ocho realizaron collage (cinco hombres y tres mujeres) cinco realizaron dibujos (cuatro mujeres y un hombre) y un participante realizó una figura de origami.

Entre Septiembre de 2014 y Noviembre de 2015 se realizaron 26 encuentros con los 14 participantes (dos encuentros con 12 participantes), que duraron entre 50 minutos y dos horas (con un promedio de 1:25') en lugares y horarios convenientes para los participantes, que contaran con las condiciones necesarias de silencio y que permitieran trabajar con los materiales artísticos. Cada encuentro fue transcrito textualmente y transcurrieron entre uno y dos meses entre encuentros, dependiendo de la disponibilidad de los participantes quienes, durante ese período, recibieron la transcripción correspondiente a la primera reunión. Durante los encuentros, siguiendo las recomendaciones de Bertaux (1999), la actitud fue mayormente no-directiva, atenta y de comprensión empática (Cornejo, Mendoza, \& Rojas, 2008), limitándose las preguntas a la clarificación del relato, para que los participantes desplegaran sus narrativas del modo más libre posible.

Para la producción de datos, se siguieron lineamientos éticos respecto a la postura investigativa, como el resguardo de la confidencialidad, el anonimato de los participantes y el reconocimiento de la autoría de sus obras visuales. Se contó con la aprobación del Comité de Ética de la Escuela de Psicología de la Pontificia Universidad Católica de Chile.

\section{Dispositivos de Análisis de Datos}

Para triangular el análisis de los datos visuales y verbales, profundizar en la escucha de los encuentros y realizar los ajustes necesarios a estos, se desarrollaron una serie de prácticas reflexivas: reuniones de interanálisis, reuniones de supervisión, participación en un estudio dirigido sobre el trabajo de la subjetividad en investigación y desarrollo de cuaderno reflexivo y de respuestas visuales a los encuentros. Las reuniones de interanálisis se realizaron con psicólogas y arte-terapeutas para los relatos y obras visuales, respectivamente. El cuaderno reflexivo se desarrolló como un espacio donde volcar las complejidades de la escucha y de la propia participación en el proyecto (Etherington, 2004), y la respuesta visual, herramienta reflexiva proveniente del arte terapia (Fish, 2012), se utilizó para visibilizar, contener y analizar material emergente respecto a la experiencia 
de investigación difícil de expresar en términos verbales.

\section{Dispositivos de análisis de contenido}

Para los relatos de vida, siguiendo la propuesta de Sharim (2005), se realizó un análisis singular, orientado a profundizar en las particularidades de cada caso. Las transcripciones se fueron analizando por párrafo, rescatando temas, sentidos y significados emergentes en los relatos, en el modo que sugiere Smith (2004) respecto al análisis interpretativo fenomenológico. También se fueron consignando hitos y aspectos clave de la ordenación temporal (Sharim, 2005). A partir de esta etapa de análisis singular, se reconstruyó un relato de vida por caso, con base en los ejes temáticos y significados más relevantes para cada participante. También se realizó un análisis singular de las obras.

Siguiendo una perspectiva fenomenológica, se consideró el relato de cada participante sobre su obra y los contenidos que se elaboraron en los diálogos al respecto a través de los dos encuentros (Betensky, 1995; Carpendale, 2011). Luego, siguiendo la propuesta de Acosta (2001), el análisis consideró la dimensión estética de las obras (materialidad, composición y colores), puestas en el contexto del relato de sus creadores y también el clima emocional que transmitía la obra. Finalmente, se consideró el impacto subjetivo y las resonancias emocionales de la obra sobre la investigadora como parte del análisis (Leclerc, 2012).

El tercer momento analítico fue de carácter transversal. Se analizaron las recurrencias y diferencias en los relatos y obras de todos los participantes considerando los contenidos, características formales, las posiciones subjetivas desde las cuales los desarrollaron y las resonancias de la escucha. Con base en este análisis, se construyeron categorías, que articularon los principales sentidos y significados que atribuyen los participantes a sus historias migratorias.

\section{Resultados}

La pluralidad de los sentidos atribuidos a la migración

Los resultados mostraron que las experiencias migratorias son significadas pluralmente, revelando la riqueza de las distintas estrategias de búsqueda de sentido. Esto, aunque la mayoría compartió experiencias clave, como "partir desde cero".

En este escenario, se identificaron ocho tácticas, entendidas como prácticas de apropiación singulares y temporales, que buscan transformar eventos en oportunidades desde una esfera ajena al poder (De Certau, 1988), o estrategias identitarias, en el sentido que proponen Camilleri y Malewska-Peyre (1997), utilizadas por los participantes para dar sentido a sus experiencias migratorias, asociadas a posiciones subjetivas diferentes. Estas incluyeron considerar la inmigración como experiencia de autoafirmación; como promotora de la individuación; como conquista de espacios de libertad y auto-determinación personal; como crisis identitaria y apertura reflexiva, como experiencia en continua construcción; como escape de un pasado doloroso; como desencanto y discriminación y como experiencia de diferencia y crítica social. En la mayoría de los casos, se encontraron elementos de cada una, aunque con distinta preeminencia.

Cada una de estas posiciones se relacionó con el grado percibido de cambio personal. Aunque los participantes consideraron que cambiar es inevitable al encontrarse con una cultura diferente, se observaron diferencias al respecto. Quienes percibieron menos cambios fueron los participantes que significaron la migración como una experiencia de auto-afirmación, que confirmó sus relatos pre-existentes sobre sí mismos y, en segunda instancia, quienes adoptaron una postura más crítica respecto a los modos de vida en Chile a través del desarrollo de prácticas para mantener una vida lo más similar posible a la que llevaban en Colombia (e.g., comiendo igual, viviendo en un barrio con alta presencia de migrantes, 
limitando su círculo de amistades a migrantes). Por otra parte, dos participantes mujeres que estaban desencantadas y habían sufrido fuerte discriminación, consideraron que el cambio había tenido un carácter negativo.

$\mathrm{Al}$ respecto, cabe destacar que, a pesar de las dificultades -salvo las dos participantes que significaron la migración como desencanto, asociado a la discriminación sufridalos participantes valoraron positivamente la migración, esto debido a que, desde su perspectiva, las ganancias superan a las dificultades. Sin embargo, reconocieron que la pregunta por volver o quedarse está en continua evaluación. Evocaron la imagen de una balanza, en la que cada grano cuenta:

Uno como que va colocando granitos en, en, en la balanza y, y eso va a ayudando de que, de que siga como, no sé si convenciéndome de que estoy bien acá, pero, pero son puntos por decirlo así a favor para seguir tranquilo acá y no decir "me devuelvo y prefiero seguir luchándola en Colombia" (Fernando, 31 años, Septiembre de 2015).

La construcción de sentido individual evoluciona dinámicamente conforme pasa el tiempo. Más que completarse de una vez, la necesidad de legitimar y apropiarse de la experiencia migratoria es proceso continuo, que sitúa a los participantes permanentemente en la disyuntiva de quedarse, volver o partir a otro lugar, incluso aunque lleven un tiempo considerable en Chile:

Te das cuenta de que si te desprendiste de padre y madre, lo demás son cosas efímeras ... el temor de salir lo tienes una sola vez, ya después no te da miedo nada. Y es lo que me pasa a mí, o sea yo he visto, yo digo "estoy en Chile" y disfruto Chile; mañana no sé (Alfonso, 33 años, Noviembre de 2015).

En un contexto en que el futuro aparece abierto -y por lo mismo, marcado por un monto importante de incertidumbre- las experiencias de logro resultan fundamentales para dar solidez al proyecto migratorio. Esto, pues la migración es mayoritariamente significada como un "reto personal" que pone a prueba las propias capacidades.

\section{La presión por el éxito}

Los participantes llegaron a Chile voluntariamente y enfatizaron que no se encontraban en una situación "desesperada" en Colombia, sino que anhelaban más: más oportunidades, más libertad, más aventura. Esta voluntariedad de su proyecto migratorio, aunque podría ser percibida como un factor facilitador, les impuso una carga importante. Sentían que había mucho en juego y que debían demostrar a sus familias, amigos y a ellos mismos que habían tomado la decisión correcta dejando Colombia.

Esto se volvió un reto personal donde no podía decir "me voy porque me quedó grande" entonces por eso ... Desde que yo llegué totalmente todo es una prueba ... iseré capaz o no seré capaz? (Pablo, 32 años, Septiembre de 2014).

La significación de la migración como "reto personal" incide también en la dificultad que los participantes revelaron para comunicar sus problemas y necesidades a otros, por temor a que tal comunicación desestabilizara la posición conquistada, que permite dar sentido a la decisión de quedarse: "ni loca decir en Colombia "necesito plata" ino! no podía permitirme eso" (Ana, 29 años Marzo de 2015).

En este contexto, aunque las redes de amigos e inmigrantes en Chile son consideradas potenciales fuentes de apoyo, los participantes tendieron a significar la resistencia, el esfuerzo y la fortaleza personal como los factores clave para "salir adelante". El fracaso y el éxito de sus proyectos migratorios son vividos como una responsabilidad inherentemente personal. Consideran que las oportunidades disponibles no valen si no existe un genuino esfuerzo por aprovecharlas.

Cuando uno quiere las cosas y salir adelante hay que ser perseverante, hay que tener fortaleza, no hay que rendirse, hay que luchar...eh, hay que ser valiente... hay momentos en que uno se 
desploma, hay que hacerlo, hay que desahogarse pero seguir...y no rendirse tan fácil iporque no es fácil! Estás en un lugar totalmente ajeno a uno, donde uno no es nadie y empezar a abrirte ese camino ... eso es como el punto, de pronto. Ser fuerte y no desistir. Si quiere uno las cosas, las puede lograr...eso (Ana, 29 años, Marzo 2015).

Los participantes significaron la experiencia migratoria como un proceso que ha desafiado sus límites y capacidades, permitiéndoles reconocer en sí mismos una fortaleza que no necesariamente conocían previamente "se da uno cuenta de que uno es un teso, o sea, de que hace esfuerzos de que uno no imaginó" (Lucía, 36 años, Julio de 2015.

Así, los participantes consideran que la migración los ha puesto en posición de "salir adelante", lo que se enmarca dentro de un mandato general compartido de orientarse al futuro y seguir avanzando, sin importar el cansancio, la enfermedad o la soledad. Las mismas frases se repiten casi textuales en sus relatos: "yo también me canso, y todo pero, yo digo "no, no me voy, pa' delante no más" (Nancy, 46 años, Julio de 2015); "obviamente hay veces que se derrumba uno, que está uno solo, pucha...pero igual uno sigue adelante" (Ana, 29 años, Marzo 2015); "siempre yo le inculco eso a mis hijos, mi mujer, siempre le digo que salga pa' delante, no más" (Manolo, 44 años, Octubre de 2015); "Hay que aceptar, seguir pa' adelante" (Camilo, 26 años, Agosto de 2015); "hay un dicho que en la costa se dice: pa' adelante es pa' allá, para atrás ni para coger impulso" (Víctor, 48 años, Septiembre de 2014); "tienes que optimizar tus recursos, salir adelante con lo que tienes" (Pablo, 32 años, Noviembre de 2014). Y así, uno a uno, los participantes van poniendo el acento en el esfuerzo personal y la orientación al futuro.

\section{El énfasis en el desarrollo y crecimiento personal}

La mayoría de los participantes significaron la migración como un proceso asociado a cambios identitarios. En sus relatos y obras, esto aparece vinculado a una serie de factores. Primero, a la experiencia de poder "reinventarse" o, incluso, "renacer". La posibilidad de partir de cero, más que atemorizante les resulta atractiva "cuando uno llega a otro país es como empezar una nueva vida también y también es maravilloso porque todo es nuevo...todo no puede ser malo" (Alejandra, 42 Octubre de 2014). Así, los participantes valoraron la apertura a nuevas formas de ser asociada a la migración:

Para mí, Chile significó un rehacerme, un reescribir mi historia ... Yo creo que el hecho de haber como, haber como vivido este proceso de, de volver a nacer...que tiene ese lado doloroso como de desarraigo, pero tiene también un lado de volver a nacer (Jaime, 34 años, Diciembre de 2014).

\section{Figura 1}

Alfonso: "Renacimiento" - $41 \times 30 \mathrm{~cm}$.

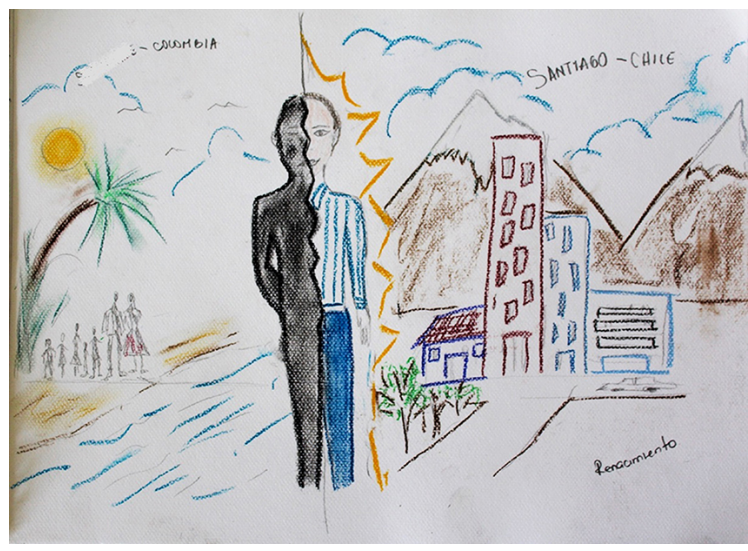

Fuente: investigación propia.

La vida me, me mostró que, aquí lo que te muestro es que... no siempre estando en el lugar ideal estás tú de ánimo para ese entorno, porque yo, esto debiera ser así al inverso, familia, playa, temperatura rica, todo lo que uno quiere, todos los elementos que tú deseaste tener en la vida no siempre están en el mismo lugar, en el mismo tiempo, en las mismas circunstancias. Yo era, yo estaba, no era que estaba, no era que estaba contra la pared ni mucho menos ino! ... eh la vida me, me dio este giro de $360^{\circ}$ y me quitó la, la presencia de mi familia física, al lado mío, pero, pero me dio, me dio otras cosas (Alfonso, 33 años, Noviembre de 2015).

La valoración de la apertura existencial no implica negar las dificultades encontradas en el proceso migratorio; más bien, los participantes 
evidenciaron el esfuerzo de encontrar sentido en las dificultades, significándolas como promotoras del desarrollo, como oportunidades de aprendizaje:

De las cosas difíciles, de las cosas buenas, de todo hay que sacarle lo bueno... el, el para qué está ocurriendo, y no un por qué, sino para qué estoy en esta circunstancia, para... qué necesito aprender ... hemos tenido que probar cosas que antes no, no, no las conocíamos, y decir: "iuy! iFui capaz! iLo logré! Eh... pude pasar esto, pude pasar estas adversidades", ahora, pienso yo, "subí otro nivel", y ya mi madurez, mi manera de ver las cosas son diferentes (Fernando, 31 años, Septiembre 2015).

Así, en la evaluación de su experiencia de desarrollo personal, los participantes identificaron dos factores clave: los mayores espacios de libertad que han ganado con la distancia y que les han permitido detenerse a reflexionar sobre sí mismos, sus trayectorias y su cultura de origen; y el haber enfrentado y superado dificultades, lo que les ha permitido descubrir una fortaleza personal mayor a la que imaginaban, y en algunos casos, mayor empatía con el dolor de otros.

Lo primero es particularmente visible en los relatos de los participantes que atribuyen un menor grado de dificultad a la experiencia migratoria y cuyas situaciones de origen resultaban restrictivas del desarrollo de su identidad: relaciones de pareja, familiares y condiciones de violencia social estrechaban sus mundos y, en contraste, la migración representa una apertura que es vivenciada como liberadora, a pesar de las dificultades.

El segundo factor es particularmente relevante, aunque no excluyente, en los relatos de quienes migraron desde condiciones de vida menos restrictivas o sentidas como menos opresoras. De este modo, el énfasis en el desarrollo personal sostiene a muchos de los participantes a través de los momentos difíciles, logrando darle sentido a la trayectoria migratoria como un aporte a su identidad. Sin embargo, es importante destacar también que, en la medida que ese desarrollo no es posible y que la experiencia migratoria pasa a ser asociada con pérdidas de aspectos del sí mismo, el proyecto pierde sentido y pasa a ser significado de un modo más negativo, tal como muestran los casos de quienes han sufrido en mayor medida experiencias de rechazo y discriminación.

\section{El rechazo a identificarse con categorías}

Pese a que los participantes reconocieron el impacto de la migración en sus vidas, mostraron rechazo a identificarse a sí mismos como inmigrantes. De hecho, hicieron un esfuerzo por destacar cómo sus historias se diferenciaban del "migrante típico" que deja su país por necesidad. Así también, mostraron sentirse poco identificados con los(as) "colombianos(as) en Chile" como grupo de referencia, aunque sí se identificaban con cualidades que consideraban propias de la cultura colombiana como la alegría, la capacidad de disfrute, la actitud más frontal y la valoración de los aspectos comunitarios de la vida (e.g. en relación con los vecinos). Asimismo, se observó que en 12 de los 14 participantes, la identificación con aspectos de la cultura colombiana ocurre a nivel más privado que público, por ejemplo, a través de la comida.

En relación con la vinculación con otros migrantes, tanto a nivel individual como colectivo, se observaron diferencias de género significativas. En su mayoría, las mujeres fueron tajantes en establecer diferencias y criticar a quienes no se "adaptan" a la sociedad chilena "si yo soy extranjera, estoy llegando acá, yo me tengo que adaptar a las condiciones y a las costumbres de Chile, isí?" (Ana, 29 años, Marzo de 2015); "Si yo vine a Chile, yo tengo que integrarme a Chile. Si esa fue mi decisión. $\mathrm{Si}$ yo vine por trabajo, por lo que sea, yo necesito integrarme, necesito funcionar en una sociedad de cualquier manera. No puedo andar chocando" (Alejandra, 42 años, Diciembre de 2014). Es relevante resaltar que al hablar de las "costumbres de Chile" las participantes refieren fundamentalmente a la relevancia que observan que tiene la noción de orden, y la expectativa que los migrantes actúen en concordancia. Así también, la expectativa de mantener un "bajo 
perfil" responde a la evitación de potenciales experiencias de discriminación, que en el caso de las mujeres podrían ser más marcadas "a veces uno dice: no hablemos mucho para que no se den cuenta que somos colombianos" (Gracia, 42 años, Octubre de 2014).

Los hombres, en cambio, mostraron una postura menos tajante. Como las mujeres, se identificaron y valoraron aspectos que consideran característicos de la cultura colombiana, como la alegría y el trato más frontal, y expresaron la pérdida que representaba para ellos el cambio de ambiente, pero, a diferencia de ellas, establecieron menos comparaciones sociales en relación a otros "colombianos en Chile". Tampoco evitan espacios colombianos, aunque fueron explícitos en declarar que no los buscan.

La excepción a esta tendencia se observó en los dos participantes afrocolombianos (hombre y mujer), que mostraron mayor identificación con otros migrantes colombianos en Chile como grupo de referencia, y que al mismo tiempo sostuvieron una postura particularmente crítica hacia la vida social en Chile, reivindicando su derecho a mantener la propia cultura, especialmente en lo que refiere a la forma de celebrar, aunque esto pudiera generar conflictos de convivencia con los vecinos. En estos participantes, la identificación cultural mostró una dimensión racializada.

\section{Conclusiones}

En relación con las preguntas planteadas, los resultados muestran que los participantes construyen sentido de sus experiencias migratorias en modos diversos y altamente individuales, incluso quienes migraron con parejas o familia. La migración no se enmarca en un proyecto colectivo y por lo mismo, las estrategias aculturativas, las tácticas cotidianas y, en definitiva, el éxito o fracaso de la migración es significado principalmente como consecuencia del esfuerzo personal.

Esta postura es concordante con los procesos de individualización social descritos respecto a la modernidad tardía, en que "el peso de la construcción de las pautas y la responsabilidad del fracaso caen primordialmente sobre los hombros del individuo" (Bauman, 2003, p. 6). Los participantes se sienten los únicos responsables de sus vidas y eso les confiere una carga importante. La alta valoración que muestran los participantes del sentido de autonomía y agencia se manifiesta en una experiencia de conflicto interno respecto a la posibilidad de buscar ayuda ante situaciones desafiantes o abrumadoras. De hecho, en tales casos, intentaron desplegar estrategias de afrontamiento individuales, declinando visibilizar sus dificultades o solicitar ayuda institucional, prefiriendo -en última instanciabuscar apoyo entre sus amistades. Esto es consistente con lo reportado por la Fundación para la Superación de la Pobreza (FUSUPO, 2012).

Esto se relaciona también con que los participantes tendieron a articular la experiencia migratoria en su historia de vida en relación con los logros, con el desarrollo y con el descubrimiento de sus fortalezas. Los participantes emergen en sus relatos y obras como sujetos con un fuerte sentido de agencia, determinados a demostrarle a los demás y a sí mismos, su capacidad para salir adelante en casi cualquier circunstancia. En este sentido, nuestros resultados son consistentes con los de Bobowik (2013), respecto a que los inmigrantes no necesariamente experimentan un sentido de sí mismos disminuido ni se focalizan continuamente en la experiencia de pérdida de su cultura y sus relaciones. Efectivamente, los resultados mostraron que la migración instala un quiebre en la historia de vida y que tal quiebre es significado parcialmente como pérdida, pero también, como oportunidad de apertura y reflexión sobre sí mismos, como un espacio para poner en perspectiva los modos de vida pasados y proyectarse hacia el futuro, generándose oportunidades de cambio identitario.

Los participantes reconocen que la migración los ha cambiado, pero significan ese cambio de forma mayoritariamente positiva. Esto, sin negar las dificultades, pero esforzándose por 
transformarlas en oportunidades de crecimiento y aprendizaje. Es en este punto, entre la posibilidad de libertad y la experiencia de pérdida y restricciones, donde los procesos personales de construcción de sentido resultan claves para determinar la significación de la experiencia migratoria. En este espacio, la migración adquiere una dimensión existencial (Beltsiou, 2016) que evidencia que las conceptualizaciones focalizadas en una u otra dimensión (pérdida/oportunidad) resultan incompletas para poder dar cuenta de las experiencias de los migrantes.

Todo lo anterior evidencia que la construcción social de la categoría "inmigrante" requiere reformulación, pues caracterizarlos como aquellos que dejan sus países porque "deben" hacerlo (Behar, 2013) no logra dar suficiente cuenta de la complejidad de las motivaciones y significados de las migraciones actuales. Más aún, podría argumentarse que la tendencia cultural a representar a los migrantes de forma negativa, fallando en reconocer sus fortalezas (Ainslie, 2009; Bobowik, 2013), sumada al mandato cultural actual de promover la autonomía y evitar la dependencia, podría dejar a los migrantes en una posición particularmente compleja, haciendo difícil la visibilización de las particularidades de sus experiencias y necesidades, y dificultando el acceso a los recursos disponibles, lo que obstaculiza que puedan recibir ayuda cuando la necesitan (por ejemplo, en términos de acceso a información sobre regulaciones legales y laborales).

Dadas las representaciones sociales sobre los migrantes, expresadas a través de los medios de comunicación (FUSUPO, 2012), no es sorprendente que los participantes se mostraran renuentes a identificarse a sí mismos como inmigrantes y a participar de colectividades migrantes. Guarnizo y Díaz (1999) han argumentado en -en el caso de migrantes provenientes de Colombia- la renuencia a participar de colectividades migrantes sería particularmente marcada y se asociaría al peso de condiciones históricas particulares de Colombia, que habrían contribuido a instalar cierto sentido de desconfianza, mediado por la duda respecto del pasado de otros migrantes y el temor a ser asociados con actividades ilícitas; temor que se contrarrestaría limitando los vínculos entre migrantes a familiares y amigos.

Sin embargo, la renuencia a identificarse como migrantes también puede atribuirse a características de la sociedad chilena. Según los resultados de FUSUPO (2012), un 41.1\% de los migrantes se ha sentido discriminado; resultados consistentes con la Encuesta sobre Desigualdad en Chile (PNUD, 2017), según la cual un $41 \%$ de los encuestados -chilenos y migrantes-ha sufrido discriminación por una serie de factores, incluyendo --en orden de relevancia- clase, género, lugar de residencia, vestimenta y apariencia física, y origen cultural. Estos resultados dan cuenta que en Chile existe una fuerte desigualdad en el trato social, y permiten pensar que las diferencias en las tácticas cotidianas, estrategias identitarias y aculturativas desplegadas por los participantes, que aparecen diferenciadas por género, tal como encontraron Arenas y Urzúa (2016) en su estudio, así como la reticencia a identificarse como migrantes, pueden ser maniobras para lidiar con el encuentro cultural y la diferencia en una sociedad en que las desigualdades se sienten fuertemente, y en la que inclinarse hacia la asimilación puede parecer inicialmente una táctica más segura y menos demandante. Esto, aunque los chilenos declaren valorar el multiculturalismo como estrategia preferida de relación con los migrantes (Sirlopú, Melipillán, Sánchez, \& Valdés, 2015).

Todo lo anterior apunta a la existencia de brechas entre discursos y prácticas sociales en relación con la equidad -en sentido amplioy con la valoración de la diferencia en el caso de los migrantes, en particular. Esto destaca la relevancia de que las políticas públicas migratorias consideren las experiencias subjetivas de los migrantes, así como los factores individuales al desarrollar, como estrategias de outreach.

Así, este estudio evidencia la necesidad de seguir estudiando las experiencias de los migrantes, profundizando en aspectos potencialmente diferenciadores -como generación, clase y género- para promover el 
desarrollo de políticas públicas que favorezcan el encuentro intercultural de un modo que permita abrazar la diversidad sin homologar la diferencia a la jerarquía, promoviendo formas de interacción que permitan el enriquecimiento cultural mutuo.

\section{Agradecimientos}

Este artículo forma parte de la tesis "Identidad y Migración: Tensiones y Oportunidades en la Articulación de una Historia Personal" del Doctorado en Psicología de la Pontificia Universidad Católica de Chile. Se agradece el apoyo económico de la Comisión Nacional de Investigación Científica y Tecnológica CONICYT y del proyecto CONICYT/ FONDAP/15130009.

\section{Referencias}

Acosta, I. (2001). Rediscovering the dynamic properties inherent in art. American Journal of Art Therapy, 31, 93-97.

Ainslie, R. C. (2009). Regression in the construction of the immigrant other. Psychoanalysis, Culture $\mathcal{E}$ Society, 14(1), 49-57. http://doi.org/10.1057/pcs.2008.51

Akhtar, S. (1995). A third individuation: immigration, identity, and the psychoanalytic process. Journal of the American Psychoanalytic Association, 43(4), 1051-1084. http://doi.org/10.1177/000306 519504300406

Akhtar, S. (2014). Inmigration and acculturation. Mourning, adaptation and the next generation. New York: Rowman \& Littlefield.

Arenas, P., \& Urzúa, A. (2016). Estrategias de aculturación e identidad étnica: un estudio en migrantes sur-sur en el norte de Chile. Universitas Psychologica, 15(1), 117-128. http://doi.org/10.11144/Javeriana .upsy15-1.eaie

Arfuch, L. (2010). El espacio biográfico. Dilemas de la subjetividad contemporánea. (3a Edición). Buenos Aires: Fondo de Cultura Económica.
Bauman, Z. (2003). Modernidad Líquida. México, D.F.: Fondo de Cultura Económica.

Behar, R. (2013). Traveling heavy. A memoir in between journeys. Durnham, NC: Duke University Press.

Beltsiou, J. (2016). Seeking home in the foreign: Otherness and immigration. En J.Beltsiou (Ed.), Immigration in psychoanalysis: Locating ourselves (pp. 89-108). New York, NY: Routledge.

Berry, J. W. (2005). Acculturation: Living successfully in two cultures. International Journal of Intercultural Relations, 29(6), 697-712. http://doi.org/10.1016/j.ijintrel.2 005.07 .013

Berry, J.W. (2007). Acculturation and identity. En D. Bhugra, \& K. Bhui (Eds.), Textbook of Cultural Psychiatry (pp. 169-178). New York, NY: Cambridge University Press.

Bertaux, D. (1999). El enfoque biográfico: Su validez metodológica, sus potencialidades. Proposiciones, 29, 1-23.

Betensky, M. (1995). What do you see? Phenomenology of therapeutic art expression. London: Jessica Kingsley Publishers.

Bobowik, M. (2013). The bright side of migration. From identity management to happiness (tesis doctoral sin publicar). San Sebastián: Universidad del País Vasco.

Boulanger, G. (2004). Lot' s Wife, Cary Grant , and the American Dream\#: Psychoanalysis with Immigrants. Contemporary Psychoanalysis, 40(3), 353-372. http://doi.org/10.1080/00107530. 2004.10745836

Camilleri, C. \& Malewska-Peyre, H. (1997). Socialization and identity strategies. En J.W. Berry, Y.H. Poortinga, \& J. Pandey (Eds.), Handbook of cross-cultural psychology. Volume 2: Basic processes and human development (2nd. Edition) (pp. 42-67). Boston, MS: Allyn and Bacon.

Cano, V., \& Soffia, M. (2009). Los estudios sobre migración internacional en Chile: apuntes y comentarios para una agenda de investigación actualizada. Papeles de Población, 15(61), 129-167. 
Cárdenas, M., \& Yáñez, S. (2012). Nuevas formas de muestreo para minorías y poblaciones ocultas: Muestras por encuestado conducido en una población de inmigrantes sudamericanos. Universitas Psychologica, 11(2), 571-578.

Carpendale, M. (2011). A traveler's guide to art therapy supervision. Bloomington, IN: Trafford Publishing.

Courtis, C. (2011). Marcos institucionales, normativos y de políticas sobre migración internacional en Argentina, Chile y Ecuador. En Martínez J. (2011). Migración internacional en América Latina y el Caribe: Nuevas tendencias, nuevos enfoques. Santiago: CEPAL.

Cornejo, M. (2006). El enfoque biográfico: trayectorias, desarrollos teóricos y perspectivas. Psykhe, 15(1), 95-106. http://doi.org/10.4067/S0718-2228 2006000100008

Cornejo, M., Mendoza, F., \& Rojas, R. (2008). La investigación con relatos de vida: pistas y opciones del diseño metodológico. Psykhe, 17(1), 29-39. http://doi.org/10.4067/S0718 $-22282008000100004$

De Certau, M. (1988). The practice of everyday life. Berkeley, CA: University of California Press.

De Fina, A. (2003). Identity in narrative: A study of immigrant discourse. Filadelfia, PA: John Benjamins Publishing Company.

De Gaulejac, V. (1996). Historia de vida y sociología clínica. Proposiciones, 29. Santiago de Chile: Ediciones Sur. Recuperado de http://www.sitiosur.cl/r.php $? \mathrm{id}=750$

Doña Reveco, C., \& Mullan, B. (2014). Migration Policy and Development in Chile. International Migration, 52(5), 1-14. http://doi.org/10.1111/imig.12157

Esses, V. M., Deaux, K., Lalonde, R. N., \& Brown, R. (2010). Psychological Perspectives on Immigration. Journal of Social Issues, 66(4), 635-647. http://doi.org/10.1111/j.1540-456 0.2010.01667.x
Etherington, K. (2004). Becoming a reflexive researcher. Using ourselves in research. London: Jessica Kingsley Publishers.

Fish, B. J. (2012). Response Art: The Art of the Art Therapist. Art Therapy, 29(3), 138-143. http://doi.org/10.1080/07421656. 2012.701594

Flick, U. (2004). Introducción a la investigación cualitativa. Madrid: Editorial Morata.

Fundación para la Superación de la Pobreza y Escuela de Periodismo Universidad Diego Portales [FUSUPO]. (2012). Ser migrante en el Chile de hoy. Recuperado de http://www.comunicacionypobreza.cl/w p-content/uploads/SER-MIGRANTE-ENEL-CHILE-DE-HOY.pdf

González, F. (2016). Only what is human can truly be foreign. The trope of immigration as a creative force in psychoanalysis. En J. Beltsiou (Ed.), Immigration in psychoanalysis: Locating ourselves (pp. 15-38). New York: Routledge.

Grinberg, L., \& Grinberg, R. (1996). Migración y exilio. Estudio psicoanalítico. Madrid: Biblioteca Nueva.

Guarnizo, L. E., \& Diaz, L. M. (1999). Transnational migration: a view from Colombia. Ethnic and Racial Studies, 22(2), 397-421. http://doi.org/10.1080/01419879 9329530

Hermans, H. J. M. (2001). The dialogical self: Toward a theory of personal and cultural positioning. Culture $\mathcal{E}$ Psychology, 7(3), 243-281. http://doi.org/10.1177/1354067X 0173001

Leclerc, J. (2012) When the image strikes. Postmodern thinking and epistemology in art therapy. In H. Burt (Ed.), Art therapy and postmodernism. Creative healing through a prism (pp. 367-378). Filadelfia, PA: Jessica Kingsley Publishers.

Lijtmaer, R. M. (2001). Splitting and Nostalgia in Recent Immigrants: Psychodynamic Considerations Review of the Literature. Journal of American Academy of Psychoanalysis and Dynamic Psychiatry, 29(3), 427-438. 
Lipovetsky, G. \& Charles, S. (2006). Los tiempos hipermodernos. Barcelona: Anagrama

Lobban, G. (2006). Immigration and dissociation. Psychoanalytic Perspectives, 3(2), 73-91. http://doi.org/10.1080/155180 6X.2006.10472954

Martínez, J. (2005). Migración internacional: iSe puede hacer algo que favorezca a los países de América Latina y el Caribe? Praxis, 7(7) $27-43$.

Ministerio de Desarrollo Social. (2016). Encuesta de Caracterización Socioeconómica Nacional (CASEN) 2015. Recuperado de h ttp://observatorio.ministeriodesarrollosocia l.gob.cl/casen-multidimensional/casen/doc s/casen_nmigrantes_2015.pdf

Ministerio del Interior y Seguridad Pública. (2010). Informe anual departamento de extranjería y migración. Recuperado de http://www.extranjeria.gob.cl/filesapp/I nforme\%20Estimacion\%20Poblacion\%20E xtranjeros\%202008.pdf

Ministerio del interior y Seguridad Pública. (2016). Migración en Chile 2005 - 2014. Recuperado de http://www.extranjeria.gob. $\mathrm{cl}$

Moran-Ellis, J., Alexander, V., Cronin, A., Dickinson, M., Fielding, J., Sleney, J., \& Thomas, H. (2006). Triangulation and integration: Processes, claims and implications. Qualitative Research, 6(1), 45-59. http://doi.org/10.1177/1468794106 058870

Moon, C. H. (2010) (Ed.), Materials and Media in Art Therapy: Critical Understandings of Diverse Artistic Vocabularies. Florence, KY: Routledge.

Organización Internacional para las Migraciones [OIM]. (2011). Perfil Migratorio de Chile. Recuperado de http://priem.cl/wp-content/uploads/201 5/04/Stefoni_Perfil-Migratorio-de-Chile.pd $\mathrm{f}$

Organización Internacional para las Migraciones. (2013). Facts $\mathcal{E}$ Figures. Recuperado de http://www.iom.int/cms/en/sites/iom/ho me/about-migration/facts--figures-1.html
Patton, M.Q. (2002). Qualitative research $\mathcal{E}$ evaluation methods. Thousand Oaks, CA: Sage Publications, Inc.

Programa de las Naciones Unidas para el Desarrollo [PNUD] (2017). Desiguales: Orígenes, cambios y desafíos de la brecha social en Chile. Recuperado de http://www.desigu ales.org

Ravanal, M. (2005). Los grandes temas de la migración...y los de la casa (los nuestros). Praxis, 7(7) 73-79.

Reavey, P., \& Johnson, K. (2008). Visual approaches: using and interpreting images. In C. Stainton, W. Rogers, \& C. Willig (Eds.), The Sage Handbook of Qualitative Research. London: Sage.

Sharim, D. (2001). Los relatos de vida como herramienta para la investigación y formación clínica. Psykhe, 10(2), 71-76.

Sharim, D. (2005). La identidad de género en tiempos de Cambio: Una aproximación desde los relatos de vida. Psykhe, 14(2), 19-32. http://doi.org/10.4067/S0718-22282 005000200002

Sirlopú, D., Melipillán, R., Sánchez, A., \& Valdés, C. (2015). i Malos Para Aceptar la Diversidad\#? Predictores SocioDemográficos y Psicológicos de las Actitudes Hacia el Multiculturalismo en Chile. Psykhe, 24(2), 1-13. http://doi.org/10 .7764 /psykhe.24.2.714

Soler, L. (2004). Dinámica reciente de la migración intrarregional. Los colombianos en Santiago de Chile (1990-2003) (tesis de magíster sin publicar). Santiago: Universidad Alberto Hurtado.

Solimano, A., \& Tokman, V. (2007). Migraciones Internacionales en un Contexto de Crecimiento Económico: El Caso de Chile. Recuperado de http://www.eclac.org/celade/noticias/ paginas/7/29527/victortokman.pdf

Smith, J. (2004). Reflecting on the development of interpretative phenomenological analysis and its contribution to qualitative research in psychology. Qualitative Research in Psychology, 1(1), 39-54. http://doi.org/10.11 91/1478088704qp004oa 
Volkan, V. (2004). After the Violence\#: The internal world and linking objects of a refugee family. In B. Sklarew, S. Twemlow, \& S. Wilkinson (Eds.), Analysts in the trenches. Streets, schools, war zones (pp. 77-102). Hillsdale, NJ: The Analytic Press.

Weber, S. (2008). Visual images in research. In J.G Knowles \& A.L. Cole (Eds.), Handbook of the arts in qualitative research: Perspectives, methodologies, examples and issues (pp. 4153). Los Angeles, CA: Sage Publications.

\section{Notas}

* Artículo de investigación 